\title{
End-of-life care: Updates for busy clinicians
}

Two years ago, Clinical Medicine reported on the publication of NICE guideline NG31 Care of dying adults in the last days of life. ${ }^{1,2}$ This document remains invaluable as an evidence-based resource for non-specialists caring for patients in their last hours to days of life. It describes how to recognise when a patient enters their last days of life, as well as providing guidance on communication with a view to shared decision making and individualised care. It provides a balanced discussion of the complex and sometimes emotional issue of maintaining hydration, while offering basic practical advice for managing common symptoms at the end of life. As with any concise guidance, it signposts onward to further sources of education and information.

I have been delighted to serve as guest editor for this issue, focusing on improving end-of-life care. We have attempted to expand upon some of those topics most likely to be relevant to the practising physician, including updates on symptom management ${ }^{3}$ and an overview of cancer pain management. ${ }^{4}$ These are likely to be of interest even to those who feel confident with the basic concepts involved. We are reminded of the importance of collaboration and maintaining good communications with our primary care colleagues, ${ }^{5}$ and the webs of caring and support available within communities. ${ }^{6}$

Although religious and spiritual aspects of our patients' lives are often considered a personal matter, those beliefs frequently come to the forefront for dying patients and their families. It is for this reason that we are pleased to be able to include an article outlining basic beliefs of some of the UK's most common religions and how these may impact on patients' care needs and preferences at the end of life. ${ }^{7}$ This is by no means an exhaustive piece likely to be found in a theological journal; in reality, beliefs will vary and no article can replace individualised assessment and discussion. This piece is intended to provide active clinicians with the background religious knowledge needed to meaningfully participate in conversations with patients about their religious and spiritual needs. It has been suggested that this simple material be considered for inclusion within undergraduate or postgraduate curricula; a potentially controversial proposal, considering how full those curricula already are, but one that may remind us of its importance at the end of life.
As a palliative medicine trainee, I work with a variety of acute care, medical and surgical colleagues, providing advice, clinical care and education in this area; it is clear to me that providing high quality end-of-life care is truly the responsibility of everyone involved. This spans professional and clinical boundaries, primary and secondary care, community and social support structures, and managerial hierarchies. Therefore, while this edition of Clinical Medicine examines the more clinical and practical aspects of individual patient care, we also recommend the corresponding articles in the Future Healthcare Journal and Commentary. Together, we hope these articles combine to represent a comprehensive vision of how we can all help to improve the quality of end-of-life care.

\section{References}

1 Hodgkinson S, Ruegger J, Field-Smith A et al. Care of the dying adults in the last days of life. Clin Med 2016;16:254-8.

2 National Institute for Health and Care Excellence. Clinical guidelines for care of the dying adult in the last days of life (NG31). London: NICE, 2015.

3 Star A, Boland J. Updates in palliative care - recent advancements in the pharmacological management of symptoms. Clin Med 2018;18:11-6.

4 Wood H, Dickman A, Star A, Boland J. An update in palliative care: overview and recent advancements in the pharmacological management of cancer pain. Clin Med 2018;18:17-22.

5 Millington-Sanders C. Passing the baton of trust: the Daffodil Standards. Clin Med 2018;18:9-10.

6 Abel J. Compassionate communities and end-of-life care. Clin Med 2018;18:6-8.

7 Choudry M, Latif A, Warburton KG. An overview of the spiritual importances of end-of-life care among the five major faiths of the United Kingdom. Clin Med 2018;18:23-31.

Angela Star Address for correspondence: Angela Star, Macmillan Palliative Care Unit, Northern General Hospital, Sheffield Teaching Hospitals, Herries Road, Sheffield S5 7AU, UK. Email: angela.star@sth.nhs.uk 